\title{
Canonical quantization of motion on submanifolds
}

\author{
Alexey V. Golovnev \\ Arnold Sommerfeld Center for Theoretical Physics \\ Ludwig-Maximilians University
}

Theresienstr. 37, Munich, 80333 Germany

Alexey.Golovnev@physik.uni-muenchen.de

\begin{abstract}
This is an extended version of the talk given by the Author at the 40th Symposium on Mathematical Physics held in Torun, Poland, June $25-28,2008$. We review the methods of canonical quantization of free particle motion on curved submanifolds considered as a system with second class constraints. The work is based on our previous articles, 1] and 2]. However, some new results are also presented.
\end{abstract}

\section{Introduction}

We consider the problem of quantum motion in curved spaces. It is wellknown that in the case of Euclidean spaces the correct quantum Hamiltonian is $\hat{H}=-\frac{\hbar^{2}}{2} \Delta$. Podolsky [3] in 1928 proposed that for arbitrary spaces it should be replaced by $\hat{H}=-\frac{\hbar^{2}}{2} \Delta_{L B}$ with $\Delta_{L B}$ being the Laplace-Beltrami operator. This postulate is a direct and geometrically clear generalization of the dynamics in Euclidean spaces. But if one wants to get the theory by some canonical procedure, he encounters a severe problem. For any given classical theory there is an infinite number of quantum theories with a proper $\hbar \rightarrow 0$ limit. Quantization is not unique. In Euclidean spaces Dirac recipe in Cartesian coordinates yields experimentally correct result for the theories which we usually have in theoretical physics. However, in curved spaces we do not have a notion of Cartesian coordinates and can't make a choice of the theory in this way. Of course, if the theory posesses a large enough symmetry it can be sometimes completely defined by the symmetry requirements directly at the quantum level, see [4. Nevertheless, we think it is very instructive to study the properties of classical quantization methods for constrained systems since theories with constraints are so important in modern physics. In particular, a possible solution for our problem is to embed the space under consideration into some Euclidean space and to quantize the new theory as a theory with second class constraints. As 
we shall see below, the results depend both on the choice of embedding and on the method of quantization.

In Section 2 we describe the Dirac approach in the case of codimension 1 surfaces first, give an explicit operator realization of it and show that the Dirac procedure is ambiguous. The quantum Hamiltonian depends even on a particular form in which the equation of surface is presented; a natural geometric way of fixing this freedom is explained. We also compare these results [1] with those obtained by general relativity inspired methods in [5] and discuss the situation in higher codimensions.

In Section 3 we present the method of converting the second class constraints into the first class ones by adding some new degrees of freedom. An error contained in 1 is corrected.

In Section 4 we review the thin layer quantization. In this approach a particle moves between two equidistant infinite potential walls $[\underline{6}$. or it is subject to some potential force which in a proper limit makes it moving strictly along the surface 7. In higher codimensions the method becomes quite involved and technical, but it also exhibits interesting features [8] related to a remarkable appearance of nontrivial gauge structures (for quantum motion in submanifolds with non-flat normal bundles) first reported in [39, 40].

\section{Dirac quantization}

Suppose we have a theory with $2 N$ constraints $\phi_{a}, a=1,2, \ldots, 2 N$. These constraints are said to be of the second class if $\operatorname{det}\left\{\phi_{a}, \phi_{b}\right\} \neq 0$ even in a weak sense 9] in Dirac terminology (on the constrained surface $\phi_{a}=0, \forall a$ ). In particular, it means that, unlike the first class constraints, they do not form a closed algebra with respect to the Poisson brackets. In such a situation the standard replacement of Poisson brackets by commutators doesn't work as it would contradict $\left\{\phi_{a}, \phi_{b}\right\} \neq 0$ inequality, and more complicated procedures are needed. One possible way out is to introduce the Dirac brackets:

$$
\{f, g\}_{\mathcal{D}}=\{f, g\}-\sum_{a=1}^{2 N} \sum_{b=1}^{2 N}\left\{f, \phi_{a}\right\} \Delta_{a b}\left\{\phi_{b}, g\right\},
$$

where $\Delta_{a b}$ is the matrix inverse of $\left\{\phi_{a}, \phi_{b}\right\}$. Now $\left\{\phi_{1}, \phi_{2}\right\}_{D}=0$ and we can introduce the commutators in a usual way. Dirac bracket is degenerate and does not define any symplectic manifold but it can be regarded as a Poisson structure obtained by factorization of original Poisson bracket algebra over motions in unphysical direction [1, see the expression (7) for the momenta operators below.

As a simplest example, one can consider a free particle motion on $(n-1)$ dimensional sphere, $\sum_{i=1}^{n} x_{i}^{2}=R^{2}$, in $n$-dimensional Euclidean space. It can be considered as a system with two second class $\left(\left\{\phi_{1}, \phi_{2}\right\}=2 \vec{x}^{2} \neq 0\right)$ constraints 
[10]

$$
\begin{gathered}
\phi_{1} \equiv \sum_{i=1}^{n} x_{i}^{2}-R^{2}=0, \\
\phi_{2} \equiv \sum_{i=1}^{n} x_{i} p_{i}=0
\end{gathered}
$$

where $p_{i}$ are canonical momenta. We impose the $\phi_{1}$ condition as a primary constraint directly at the Hamiltonian level. In the Lagrange formulation we also have a Lagrange multiplyer which would generate one more pair of second class constraints (vanishing of its canonical momentum as a primary constraint and some consistency condition involving the multiplyer itself). For the physical phase space it influences neither the Poisson structure nor the physical Hamiltonian [11. Thus we prefer not to increase the number of unphysical variables more than needed and work purely within the Hamiltonian mechanics. (Note that every new constraint enters the Dirac generalized Hamiltonian multiplied by its own new arbitrary function of time 9 .)

A natural question to be asked at this point is whether one could quantize the theory without any classical rearrangement before and then impose the constraint $\phi_{1}$ at the quantum level. It would be problematic because the secondary constraint $\phi_{2}$ (i.e. consistency condition concerning the time derivative of $\phi_{1}$ ) shows up which has a non-vanishing Poisson bracket with $\phi_{1}$. The problem would be to find a natural selfadjoint restriction of the Hamiltonian to the physical space. It is possible in principle but complicated and requires quite some accuracy. We refer the reader to the Ref. [12] and proceed with the Dirac method.

A simple calculation according to (1) shows that [10]

$$
\begin{gathered}
\left\{x_{i}, x_{j}\right\}_{\mathcal{D}}=0, \\
\left\{x_{i}, p_{j}\right\}_{\mathcal{D}}=\delta_{i j}-\frac{x_{i} x_{j}}{\vec{x}^{2}}, \\
\left\{p_{i}, p_{j}\right\}_{\mathcal{D}}=\frac{1}{\vec{x}^{2}}\left(p_{i} x_{j}-p_{j} x_{i}\right) .
\end{gathered}
$$

This algebra can be satisfied by very simple (usual) coordinate operators $\hat{x}_{i}=$ $x_{i} \hat{I}$ and standard differential operators of momenta from which the normal differentiation is subtracted [1:

$$
-i \hbar \vec{\nabla} \longrightarrow-i \hbar\left(\vec{\nabla}-\frac{\vec{x}}{|\vec{x}|}\left(\frac{\vec{x}}{|\vec{x}|} \cdot \vec{\nabla}\right)\right) \equiv \hat{\vec{p}} .
$$

The problem is that $\hat{p}_{i}$ are not selfadjoint. But at the sacrifice of Leibnitz rule we can introduce new selfadjoint momenta:

$$
\hat{\tilde{p}}_{i}=\frac{1}{2}\left(\hat{p}_{i}+\hat{p}_{i}^{\dagger}\right)=\hat{p}_{i}+i \hbar \frac{n-1}{2} \cdot \frac{x_{i}}{\vec{x}^{2}} \hat{I} .
$$


The primary constraint (2) defines the space of physical states and the secondary one (3) acquires the form of identity $\hat{\phi}_{2}=\sum_{i=1}^{n}\left(\hat{x}_{i} \hat{\tilde{p}}_{i}+\left(\hat{x}_{i} \hat{\tilde{p}}_{i}\right)^{\dagger}\right) \equiv 0$. The resulting Hamiltonian [1]

$$
\hat{H}^{(\mathcal{D})} \equiv \frac{1}{2} \sum_{i=1}^{n} \hat{\tilde{p}}_{i}^{2}=-\frac{\hbar^{2}}{2} \Delta_{L B}+\frac{\hbar^{2}(n-1)^{2}}{8 R^{2}}
$$

contains a typical quantum potential $V_{q}^{(\mathcal{D})}=\frac{\hbar^{2}(n-1)^{2}}{8 R^{2}}$. We should stress that this result can be obtained in a purely algebraic manner without any kind of explicit operator realization [10.

Note also that the same procedure may lead [1] to Podolsky theory if one takes our initial definition (7) for $\hat{p}_{i}$ and Hamiltonian $\hat{H}^{(\mathcal{P})}=\frac{1}{2} \sum_{i=1}^{n} \hat{p}_{i}^{\dagger} p_{i}$ which equals $-\frac{\hbar^{2}}{2} \Delta_{L B}$ for the physical sector functions. The quantum potential is zero: $V_{q}^{(\mathcal{P})}=0$. Thus one preserves an important property of momenta operators, the Leibnitz rule, so that they are differentiations on the algebra of smooth functions. These operators are not selfadjoint and can't represent observables. But in any case they do not have any clear physical meaning being projections of generators of motions along the coordinate lines of n-dimensional flat space, which are somewhat esoteric for an observer living on the sphere. Natural observables on the sphere are generators of $S O(n)$ rotations, and they are selfadjoint (proportional to $i\left[\hat{p}_{i}, \hat{p}_{j}\right]$ ).

\subsection{Arbitrary codimension 1 surfaces}

The free motion on a codimension 1 surface $f(x)=0$ can be obtained [11] by an obvious modification of (22) and (3):

$$
\begin{gathered}
\phi_{1} \equiv f(x)=0, \\
\phi_{2} \equiv \sum_{i=1}^{n}\left(\partial_{i} f\right) p_{i}=0 .
\end{gathered}
$$

If $|\vec{\nabla} f| \neq 0$ at the physical surface (as we assume throughout the paper), these constraints are of the second class because $\left\{\phi_{1}, \phi_{2}\right\}=(\vec{\nabla} f)^{2}$. Again we introduce the Dirac brackets by the prescription (1) and get

$$
\begin{gathered}
\left\{x_{i}, x_{j}\right\}_{\mathcal{D}}=0 \\
\left\{x_{i}, p_{j}\right\}_{\mathcal{D}}=\delta_{i j}-\frac{\left(\partial_{i} f\right)\left(\partial_{j} f\right)}{(\vec{\nabla} f)^{2}}, \\
\left\{p_{i}, p_{j}\right\}_{\mathcal{D}}=\frac{1}{(\vec{\nabla} f)^{2}} \sum_{k=1}^{n}\left(\left(\partial_{j} f\right)\left(\partial_{i k}^{2} f\right)-\left(\partial_{i} f\right)\left(\partial_{j k}^{2} f\right)\right) p_{k} .
\end{gathered}
$$


One can use the following operators [1] for the quantum description: $\hat{x}_{i}=x_{i} \hat{I}$,

$$
\hat{p}_{i}=-i \hbar\left(\frac{\partial}{\partial x_{i}}-\frac{\left(\partial_{i} f\right)}{|\vec{\nabla} f|} \sum_{j=1}^{n} \frac{\left(\partial_{j} f\right)}{|\vec{\nabla} f|} \frac{\partial}{\partial x_{j}}\right)
$$

as non-selfadjoint momenta and

$$
\hat{\tilde{p}}_{i}=\hat{p}_{i}+\frac{i \hbar}{2} \sum_{j=1}^{n}\left(\frac{\partial}{\partial x_{j}}\left(\frac{\left(\partial_{i} f\right)\left(\partial_{j} f\right)}{(\vec{\nabla} f)^{2}}\right)\right)
$$

for the selfadjoint counterparts. (Here we implement the factorization over unphysical motions again.) The operator ordering problem is relevant only for the momenta commutators, and it is solved by our explicit choice of the operators as follows:

$$
\begin{gathered}
{\left[\hat{p}_{i}, \hat{p}_{j}\right]=\frac{i \hbar}{(\vec{\nabla} f)^{2}} \sum_{k=1}^{n}\left(\left(\partial_{j} f\right)\left(\partial_{i k}^{2} f\right)-\left(\partial_{i} f\right)\left(\partial_{j k}^{2} f\right)\right) \hat{p}_{k} ;} \\
{\left[\hat{\tilde{p}}_{i}, \hat{\tilde{p}}_{j}\right]=\frac{i \hbar}{2} \sum_{k=1}^{n}\left(\frac{\left(\partial_{j} f\right)\left(\partial_{i k}^{2} f\right)-\left(\partial_{i} f\right)\left(\partial_{j k}^{2} f\right)}{(\vec{\nabla} f)^{2}} \hat{\tilde{p}}_{k}+\hat{\tilde{p}}_{k} \frac{\left(\partial_{j} f\right)\left(\partial_{i k}^{2} f\right)-\left(\partial_{i} f\right)\left(\partial_{j k}^{2} f\right)}{(\vec{\nabla} f)^{2}}\right) .}
\end{gathered}
$$

We have the identity $\sum_{i=1}^{n}\left(\partial_{i} f\right) \hat{p}_{i} \equiv 0 \quad$ or $\sum_{i=1}^{n}\left(\left(\partial_{i} f\right) \hat{\tilde{p}}_{i}+\hat{\tilde{p}}_{i}\left(\partial_{i} f\right)\right) \equiv 0$ for the secondary constraint. And the physical sector is defined by the primary one: $\Psi_{\text {phys }}=\psi(x) \delta(f(x))$.

For non-selfadjoint momenta the Hamiltonian reads

$$
\hat{H}^{(\mathcal{P})}=\frac{1}{2} \sum_{i=1}^{n} \hat{p}_{i}^{\dagger} \hat{p}_{i}=-\frac{\hbar^{2}}{2}\left(\tilde{\Delta}-\left(\frac{\partial}{\partial \vec{n}}\right)^{2}-\operatorname{div}(\vec{n}) \cdot \frac{\partial}{\partial \vec{n}}\right)
$$

where $\tilde{\Delta}$ is the Laplace operator in the Euclidean space and $\vec{n}=\frac{\vec{\nabla} f}{|\vec{\nabla} f|}$ is a unit vector normal to the surface (8). In the selfadjoint case the Hamiltonian $\hat{H}^{(\mathcal{D})}=\frac{1}{2} \sum_{i=1}^{n} \hat{\tilde{p}}_{i}^{2}=\hat{H}^{(\mathcal{P})}+V_{q}^{(\mathcal{D})}(x)$ contains also a quantum potential

$$
\begin{aligned}
V_{q}^{(\mathcal{D})}= & -\frac{\hbar^{2}}{8} \sum_{i=1}^{n}\left(\sum_{j=1}^{n} \frac{\partial}{\partial x_{j}} \frac{\left(\partial_{i} f\right)\left(\partial_{j} f\right)}{(\vec{\nabla} f)^{2}}\right)^{2}+ \\
& +\frac{\hbar^{2}}{4} \sum_{i=1}^{n}\left(\frac{\partial}{\partial x_{i}}-\sum_{k=1}^{n} \frac{\left(\partial_{i} f\right)\left(\partial_{k} f\right)}{(\vec{\nabla} f)^{2}} \frac{\partial}{\partial x_{k}}\right)\left(\sum_{j=1}^{n} \frac{\partial}{\partial x_{j}} \frac{\left(\partial_{j} f\right)\left(\partial_{i} f\right)}{(\vec{\nabla} f)^{2}}\right) .
\end{aligned}
$$


Some details of rather straightforward calculations can be found in [1]. Note that in terms of the normal vectors $\vec{n}=\frac{\vec{\nabla} f}{|\vec{\nabla} f|}$ one can easily write the potential (14) down in the following form:

$$
V_{q}^{(\mathcal{D})}=\frac{\hbar^{2}}{4}\left(\frac{1}{2}\left(\sum_{i} \partial_{i} n_{i}\right)^{2}+\sum_{i, k} \partial_{i}\left(n_{k} \partial_{k} n_{i}\right)+\frac{1}{2} \sum_{i, k, m} n_{i} n_{k} n_{m} \partial_{k m}^{2} n_{i}\right)
$$

using the obvious relations $\sum_{i} n_{i} \partial_{k} n_{i}=0$ and $\sum_{i} n_{i} \partial_{k m}^{2} n_{i}=-\sum_{i}\left(\partial_{m} n_{i}\right)\left(\partial_{k} n_{i}\right)$.

Unfortunately both Hamiltonians, $\hat{H}^{(\mathcal{D})}$ and $\hat{H}^{(\mathcal{P})}$, are ambiguous; they take different values for those functions which represent one and the same surface. (And the problem exists even for spheres.) Indeed, any surface can be represented by its tangent paraboloid at some point: $f(y)=y_{n}-\frac{1}{2} \sum_{\alpha=1}^{n-1} k_{\alpha} y_{\alpha}^{2}+\mathcal{O}\left(y_{\alpha}^{3}\right)$ where $y_{\alpha}$ are Cartesian coordinates. (It is not a priori obvious that this accuracy is enough for calculating the quantum potential but one can easily check that in this case it is, see [1.) Then (14) gives

$$
V_{q}=\frac{\hbar^{2}}{8}\left(\left(\sum_{\alpha=1}^{n-1} k_{\alpha}\right)^{2}+2 \sum_{\alpha=1}^{n-1} k_{\alpha}^{2}\right)+\mathcal{O}\left(y_{\alpha}\right)
$$

in the vicinity of the point $\vec{y}=0$. For a sphere the principal curvatures are $k_{\alpha}=\frac{1}{R}$ and at the chosen point we have $V_{q}=\frac{\hbar\left(n^{2}-1\right)}{8 R^{2}}$ which differs from our previous result (and that of [10]). So, the Dirac recipe is ambiguous. To fix the freedom, we propose the following (geometrically natural) choice of the function $f(x)$ : up to the sign it should be equal to (some function of) the distance from the surface $f=0$. After that we have $|\vec{\nabla} f|=1$ and $\partial_{i} n_{k}=\partial_{k} n_{i}$, $\sum_{k} n_{k} \partial_{k} n_{i}=0$ where $n_{k}=\partial_{k} f$. Then a simple calculation [1] shows that the quantum potential on the $f(x)=0$ surface is $V_{q}^{(\mathcal{D})}=\frac{\hbar^{2}}{8}\left(\sum_{\alpha=1}^{n-1} k_{\alpha}\right)^{2}$. For spheres it yields the previous result. The kinetic part of the Hamiltonians for our choice of unit normals

$$
\tilde{\Delta}-\left(\frac{\partial}{\partial \vec{n}}\right)^{2}-\operatorname{div}(\vec{n}) \cdot \frac{\partial}{\partial \vec{n}}=\Delta_{L B}
$$

equals to the Laplace-Beltrami operator on the physical surface [1]. But in the general case (when the unit normal vector $\vec{n}=\frac{\vec{\nabla} f}{|\vec{\nabla} f|}$ would not be orthogonal to the surfaces $f(x)=$ const $\neq 0$ ) this result would not be true. The vector $\vec{n}$ will change its direction while moving apart from the initial surface, and the second normal derivative $\left(\frac{\partial}{\partial \vec{n}}\right)^{2}$ would add some extra (first order differential) term to $\Delta_{L B}$.

Let's consider a simple illustration. For a circle in a plane we would use $f(x, y)=|y|-\sqrt{1-x^{2}}$ (instead of $\left.f(x, y)=x^{2}+y^{2}-1\right)$ and approximate it 
near the $(x, y)=(0,-1)$ point by a parabola $f(x)=y-\frac{x^{2}}{2}+1=0$. We have $n_{x}=-\frac{x}{\sqrt{1+x^{2}}}$ and $n_{y}=\frac{1}{\sqrt{1+x^{2}}}$. The selfadjoint momenta can be easily found as

$$
\begin{gathered}
\hat{p}_{x}=-\frac{i \hbar}{1+x^{2}}\left(\frac{\partial}{\partial x}+x \frac{\partial}{\partial y}\right)+\frac{i \hbar x}{\left(1+x^{2}\right)^{2}}, \\
\hat{p}_{y}=-\frac{i \hbar x}{1+x^{2}}\left(\frac{\partial}{\partial x}+x \frac{\partial}{\partial y}\right)+\frac{i \hbar\left(x^{2}-1\right)}{2\left(1+x^{2}\right)^{2}} .
\end{gathered}
$$

It leads to the Hamiltonian

$\hat{H}^{(\mathcal{D})}=-\frac{\hbar^{2}}{2}\left(\frac{1}{1+x^{2}}\left(\frac{\partial}{\partial x}+x \frac{\partial}{\partial y}\right)^{2}-\frac{2 x}{\left(1+x^{2}\right)^{2}}\left(\frac{\partial}{\partial x}+x \frac{\partial}{\partial y}\right)\right)+\frac{\hbar^{2}\left(3-2 x^{2}-5 x^{4}\right)}{8\left(1+x^{2}\right)^{4}}$

which gives $V_{q}=\frac{3 \hbar^{2}}{8}$ for the quantum potential at $x \rightarrow 0\left(\right.$ instead of $\left.\frac{\hbar^{2}}{8}\right)$. And introducing the tangent derivative $\frac{\partial}{\partial \vec{t}}=\frac{1}{\sqrt{1+x^{2}}}\left(\frac{\partial}{\partial x}+x \frac{\partial}{\partial y}\right)$ we get

$$
\hat{H}^{(\mathcal{P})}=-\frac{\hbar^{2}}{2}\left(\Delta_{L B}-\frac{2 x}{\left(1+x^{2}\right)^{3 / 2}} \cdot \frac{\partial}{\partial \vec{t}}\right)
$$

for the kinetic energy operator with $\Delta_{L B}=\frac{\partial^{2}}{\partial \vec{t}^{2}}$.

\subsection{Relation to GR-like methods}

Some time ago this problem was tackled in Ref. [5] by methods typical to general relativity. The quantization was performed in Cartesian coordinates but a curvilinear coordinate system was also used. One of the coordinates $q_{0}$ was chosen to be the value of the function $f$ and the others had to be orthogonal to it. Then the authors of [5] defined the curvilinear momenta $\hat{p}_{\mu}$ in terms of Cartesian ones. The Cartesian commutators (obtained from Dirac brackets) implied the commutation relations for the curvilinear operators $\left[q^{\nu}, \hat{p}_{\mu}\right]=i \hbar\left(\delta_{\mu}^{\nu}-n^{\nu} n_{\mu}\right)$ and set $p_{0}=0$. After doing this one can arrive at $[5]$

$$
\hat{H}^{(\mathcal{D})}=\frac{1}{2} \sum_{i, j=1}^{n-1} g^{-1 / 4} \hat{p}_{i} g^{1 / 2} g^{i j} \hat{p}_{j} g^{-1 / 4}+V_{q}
$$

where $g_{i j}$ is the metric on the physical surface and the quantum potential is equal to our result for the special choice of the equation of surface, $\frac{\hbar^{2}}{8}(\operatorname{div} \vec{n})^{2}$.

The authors interpreted the kinetic part of the Hamiltonian as the LaplaceBeltrami operator on the hypersurface $f=0$. It would indeed be true if the momenta were the standard ones for the hypersurface, but they were defined to be symmetric in the ambient space which is not the same. Up to the setting the normal differentiation to zero, they would be the standard symmetric momenta operators for the curvilinear coordinate system in the ambient Euclidean space. It amounts to the difference between $\frac{1}{g^{1 / 4}} \partial_{i} g^{1 / 4}$ and 
$\frac{\sqrt{|\nabla f|}}{g^{1 / 4}} \partial_{i} \frac{g^{1 / 4}}{\sqrt{|\nabla f|}}$. If the function $f$ depends only on the distance from the surface $\left(\partial_{i}|\nabla f|=0\right)$ then it makes nothing and our results coincide. What changes if we have another function $f$ ? A linear differential operator $\frac{\hbar^{2}}{2} \sum_{i, j} \frac{\partial_{i}|\nabla f|}{|\nabla f|} g^{i j} \partial_{j}$ gets added to $-\frac{\hbar^{2}}{4} \Delta_{L B}$ and two additional terms appear in the quantum potential: $-\frac{\hbar^{2}}{8} \cdot \frac{(\vec{\nabla}(|\nabla f|))^{2}}{(\nabla f)^{2}}+\frac{\hbar^{2}}{4} \operatorname{div} \frac{\vec{\nabla}(|\nabla f|)}{|\nabla f|}$. All the operations are related to the hypersurface $f=0$. In particular, if the gradients are taken in some Cartesian coordinates of the ambient space, the differentiations should be projected to the hypersurface. In this coordinates the vector $\frac{\vec{\nabla}(|\nabla f|)}{|\nabla f|}$ in the tangent space of $f=0$ would have the following components: $\frac{1}{|\nabla f|}\left(\partial_{i}-n_{i} \sum_{k} n_{k} \partial_{k}\right)|\nabla f|=$ $\sum_{j} \frac{\partial_{j} f}{|\nabla f|} \partial_{j} \frac{\partial_{i} f}{|\nabla f|}$. And the new terms in the quantum potential can be transformed to $\frac{\hbar^{2}}{4} \sum_{i, j} \partial_{i}\left(n_{j} \partial_{j} n_{i}\right)-\frac{\hbar^{2}}{8} \sum_{i}\left(\sum_{j} n_{j} \partial_{j} n_{i}\right)^{2}$ in a complete accordance with (15). The differential operator converts to $\sum_{i, k} n_{k}\left(\partial_{k} n_{i}\right) \partial_{i}$ in Cartesian coordinates, which is exactly what would come out of the second normal derivative $\left(\frac{\partial}{\partial \vec{n}}\right)^{2}$ in our approach.

\subsection{Dirac quantization in higher codimensions}

Going to higher codimensions (the surface $f^{(a)}=0$ for $\left.a=1,2 \ldots N\right)$ complicates the things considerably because the Poisson brackets of $\sum_{i} p_{i} \partial_{i} f^{(a)}$ and $\sum_{i} p_{i} \partial_{i} f^{(b)}$ (the secondary constraints) do not vanish even if $\vec{\nabla} f^{(a)} \cdot \vec{\nabla} f^{(b)}=0$. It affects the momenta commutators. Nevertheless, if the normal bundle of the submanifold is flat and we can (locally) choose the set of functions $f$ satisfying the conditions $\left|\vec{\nabla} f^{(a)}\right|=1$ for all $a$ and $\vec{\nabla} f^{(a)} \cdot \vec{\nabla} f^{(b)}=0$ for $a \neq b$ then quite bulky but straightforward calculations show that the generalization of the previous results is very simple:

$$
\begin{gathered}
\hat{\tilde{p}}_{i}=-i \hbar\left(\frac{\partial}{\partial x_{i}}-\sum_{a=1}^{N} n_{i}^{(a)} \sum_{j=1}^{n} n_{j}^{(a)} \cdot \frac{\partial}{\partial x_{j}}\right)+\frac{i \hbar}{2} \sum_{j=1}^{n} \frac{\partial}{\partial x_{j}}\left(\sum_{a=1}^{N} n_{i}^{(a)} n_{j}^{(a)}\right) ; \\
\hat{H}^{(\mathcal{D})}=-\frac{\hbar^{2}}{2} \Delta_{L B}+\sum_{a=1}^{N} \frac{\hbar^{2}}{8}\left(\operatorname{div} \vec{n}^{(a)}\right)^{2} .
\end{gathered}
$$

It gives the extrinsic mean curvature squared for the quantum potential.

And in the general case of higher codimensions, the straightforward approach equations become almost untractable. But the structure of terms still remains quite understandable. For example, in the case of codimension 2 one can check 
that the momentum operators can be obtained by projecting the $\frac{\partial}{\partial x_{i}}$ vectors to the subspace orthogonal to both $\vec{\nabla} f^{(1)}$ and $\vec{\nabla} f^{(2)}$ according to

$\vec{V}_{\perp}=\vec{V}-\frac{\vec{a}_{1}\left(\vec{a}_{2}\right)^{2}\left(\vec{a}_{1} \vec{V}\right)+\vec{a}_{2}\left(\vec{a}_{1}\right)^{2}\left(\vec{a}_{2} \vec{V}\right)-\left(\vec{a}_{1} \vec{a}_{2}\right)\left(\vec{a}_{2}\left(\vec{a}_{1} \vec{V}\right)+\vec{a}_{1}\left(\vec{a}_{2} \vec{V}\right)\right)}{\left(\vec{a}_{1}\right)^{2}\left(\vec{a}_{2}\right)^{2}-\left(\vec{a}_{1} \vec{a}_{2}\right)^{2}}$.

At this point the methods of previous subsection [5] become very useful. In [13] it has been shown that these methods lead to formally the same result as for the codimension 1 case (Laplace-Beltrami operator and extrinsic mean curvature squared). As it was above, the interpretation of the kinetic energy operator is correct if the normal components of the metric do not influence the momenta operators which means that $\operatorname{det}\left(\vec{\nabla} f^{(a)} \cdot \vec{\nabla} f^{(b)}\right)$ does not depend on tangential coordinates.

\section{Abelian conversion method}

Sometimes in the quantum field theory first class constraints may fail to form a closed algebra at the quantum level. Formally, they can be said to become second class upon quantization, but it is very bad for the quantum field theory with local symmetries as it corresponds to a gauge symmetry breaking. For example, this kind of anomaly occurs for the Gauss law in the model of Weyl fermions interacting with a Yang-Mills field. In [14 it was proposed to introduce some new degree of freedom in the model in order to have new constraints with the Abelian algebra and get rid of the anomaly. It resembles the appearance of the conformal factor as a new degree of freedom at the quantum level for noncritical strings 15. (Very similar situation occurs in the theory of relativistic branes 16, but it is not so clear what to make out of it in this context and also it is not known if the critical dimension exists, see [17, 18, 19] for diverse views on the subject.)

This idea can also be used in quantum mechanics with second class constraints [10]. The ("Abelian conversion") method consists of introducing new canonical pair of variables $Q, K$ and first class constraints $\sigma_{1}, \sigma_{2}:\left\{\sigma_{1}, \sigma_{2}\right\}=0$ (identically, so that the constraint algebra is Abelian) assuming $\sigma_{1}=\phi_{1}, \sigma_{2}=$ $\phi_{2}$ if $Q=0$ and $K=0$. In our case it would be $\sigma_{1}=f(x)+K$ and $\sigma_{2}=\vec{n} \cdot \vec{p}+|\vec{\nabla} f| \cdot Q$. (Note an error at this point in [1] where the $|\vec{\nabla} f|$ factor has disappeared from $\sigma_{2}$.) The next step is to find a new Hamiltonian such that $H_{S}=H$ if $Q=0$ and $K=0$ and $\left\{H_{S}, \sigma_{1}\right\}=\left\{H_{S}, \sigma_{2}\right\}=0$. The physical sector is obtained by setting $\sigma_{1}=\sigma_{2}=0$. For free motion on spheres this method gave zero quantum potential, see [10, 11. Actually, the authors of [10, 11] had the result of the form $H_{S}=H_{S}\left(\sigma_{1}, \sigma_{2}, \sum_{i<k}\left(x_{i} p_{k}-x_{k} p_{i}\right)^{2}\right)$ which 
due to relation

$$
\sum_{i<k}\left(x_{i} p_{k}-x_{k} p_{i}\right)^{2}=\left(\sum_{i} x_{i}^{2}\right)\left(\sum_{i} p_{i}^{2}-\left(\sum_{i} n_{i} p_{i}\right)^{2}\right)
$$

could be transformed to $H_{\text {phys }}=\frac{\vec{p}_{\text {phys }}^{2}}{2}$ because $\sigma_{2}^{2}=\left(\sum_{i} n_{i} p_{i}\right)^{2}$ if $Q=0$.

It was concluded that the Abelian conversion method is preferable because it involves no extrinsic geometry in the results. For this and other reasons it was used in the beautiful projection operator approach to path integral quantization of constrained systems [20] aiming at quantizing the gravity [21]. But is it possible to generalize the above result to other surfaces? In principle, methods which introduce new variables are very strong, see for example [22] and references therein. Moreover, one can proceed with a general philosophy of Abelian conversion [11] without any need of introducing such weird concepts like ghost operators 22. The question is whether it is possible to do this using the very simple geometric form of the new constraints introduced above and obtaining some relatively simple and physically sensible results. Let us search for $H_{S}=H_{S}\left(\sigma_{1}, \sigma_{2}, x, p\right)$ in the previous form

$$
H_{S}=H_{S}\left(\sigma_{1}, \sigma_{2}, g(x)\left(\sum_{i} p_{i}^{2}-\left(\sum_{i} n_{i} p_{i}\right)^{2}\right)\right)
$$

designed for getting the pure Laplace-Beltrami solution. The following relations show up:

$$
\begin{array}{r}
\left\{H_{S}, \sigma_{1}\right\}=-\sum_{i} n_{i} \frac{\partial H_{S}}{\partial p_{i}}=0, \\
\left\{H_{S}, \sigma_{2}\right\}=\sum_{i} n_{i} \frac{\partial H_{S}}{\partial x_{i}}-\sum_{i, k} p_{k}\left(\partial_{i} n_{k}\right) \frac{\partial H_{S}}{\partial p_{i}}-\sum_{i}\left(\partial_{i}|\vec{\nabla} f|\right) Q \frac{\partial H_{S}}{\partial p_{i}}=0 .
\end{array}
$$

From (18) we have

$$
\begin{aligned}
\sum_{i, k} p_{i} p_{k}\left(\sum _ { j } n _ { j } \partial _ { j } g ( x ) \left(\delta_{i k}-\right.\right. & \left.\left.n_{i} n_{k}\right)-2 g(x)\left(\partial_{i} n_{k}\right)\right)- \\
& -2 \sum_{i}\left(\partial_{i}|\vec{\nabla} f|\right) Q\left(p_{i}-n_{i} \sum_{j} n_{j} p_{j}\right)=0 .
\end{aligned}
$$

If we admit the condition $|\vec{\nabla} f|=1$ (compare with the Dirac method above) then the last term disappears and it has a non-zero solution for spheres because $\partial_{i} n_{k} \sim \delta_{i k}-n_{i} n_{k}$. But this is not true for arbitrary surfaces. Hence the result 
of [10] can't be generalized directly. Nevertheless, using a simple ansatz

$$
H_{S}=H_{S}\left(\sigma_{1}, \sigma_{2}, \sum_{i, k} C_{i k}(x) p_{i} p_{k}+\sum_{i} D_{i}(x) p_{i}+E(x)\right)
$$

with $C_{i k}=C_{k i}$ one can show [1] that in general it is possible to get a quadratic in momenta Hamiltonian (not equal to the Laplace-Beltrami operator) by this method if we admit the above definition of the function $f(x)$.

If another function is used then (18) can't be valid identically because the last term contains $Q$. But we can afford having it only in a weak sense. Then from $\sigma_{2}$ we determine $Q=-\frac{\vec{n} \cdot \vec{p}}{|\vec{\nabla} f|}$, and (18) with the ansatz (16) converts into

$$
\sum_{i, k} p_{i} p_{k}\left(\sum_{j} n_{j} \partial_{j} g(x)\left(\delta_{i k}-n_{i} n_{k}\right)+2 g(x)\left(n_{i} \sum_{j} n_{j} \partial_{j} n_{k}-\partial_{i} n_{k}\right)\right)=0
$$

which is probably not as hopeless as it was erroneously stated in [1] due to the aforementioned mistake but still can give no guarantee for the existence of a nontrivial solution. Another problem of the method is that in this setting there is no clear reason for insisting on the Abelian algebra of the first class constraints. And if we go to higher codimensions we would really need to modify the method somehow, at least by finding a more clever choice of the new constraints because, as we already mentioned, the Poisson brackets of $\sum_{i} p_{i} n_{i}^{(a)}$ and $\sum_{i} p_{i} n_{i}^{(b)}$ do not vanish even if $\vec{n}^{(a)} \cdot \vec{n}^{(b)}=0$.

\section{Thin layer quantization method}

As it was discussed in the Section 2, imposing the second class constraints directly at the quantum level is problematic. Nevertheless, we can use a more delicate procedure. We can approximate the constrained system by a motion in a thin layer around it. In quantum mechanics this approach appeared in [6, 7, for a deeper discussion see [8, 23] and [24] at the mathematical level. It can also be used for classical systems 24 and gives rise to extra potential if we take the initial conditions involving normal motions in the thin tube, but coincides with the intrinsic description if the initial velocities are tangential. However, in quantum mechanics this approach always gives a geometric potential because in the quantum realm it is impossible to eliminate the normal motion completely.

We consider $(n-1)$-dimensional smooth surface in $\mathbb{R}^{n}$ and two infinite potential walls at the distance $\delta \rightarrow 0$ from the surface. Free quantum particle moves in the thin layer of width $2 \delta$ between these potential walls. We introduce a curvilinear coordinate system in which $\left|x_{n}\right|$ equals the distance from the surface to the given point (thus playing the same role as the function $f$ in the refined approach to Dirac quantization), and the coordinate lines of $x_{1}, \ldots, x_{n-1}$ are orthogonal to that of $x_{n}$. We have the boundary condition $\left.\Psi\right|_{x_{n}=\delta}=\left.\Psi\right|_{x_{n}=-\delta}=0$ 
and Hamiltonian $\tilde{H}=-\frac{\hbar^{2}}{2} \tilde{\Delta}$ with $\tilde{\Delta}$ being the Laplace operator,

$$
\begin{gathered}
\tilde{\Delta}=\sum_{i=1}^{n} \sum_{k=1}^{n} \tilde{g}^{-1 / 2} \partial_{i} \tilde{g}^{1 / 2} \tilde{g}^{i k} \partial_{k}=\partial_{n}{ }^{2}+\left(\tilde{g}^{-1 / 2} \partial_{n} \tilde{g}^{1 / 2}\right) \partial_{n}+\Delta_{L B}, \\
\tilde{g}_{i k}=\left(\begin{array}{cc}
g_{a b} & 0 \\
0 & 1
\end{array}\right)
\end{gathered}
$$

where $\Delta_{L B}$ is the Laplace-Beltrami operator on the surface $x_{n}=$ const.

The simplest way [2] to obtain the thin layer limit is to consider the tangent paraboloid of the surface $y_{n}=\frac{1}{2} \sum_{a=1}^{n-1} k_{a} y_{a}^{2}+\mathcal{O}\left(y_{a}^{3}\right)$, where $k_{a}$ are the principal curvatures. The unit normal is $n_{a}=\frac{k_{a} y_{a}}{\sqrt{1+\sum_{a=1}^{n-1} k_{a}^{2} y_{a}^{2}}}+\mathcal{O}\left(y_{a}^{2}\right)=k_{a} y_{a}+\mathcal{O}\left(y_{a}^{2}\right)$, $n_{n}=-1+\mathcal{O}\left(y_{a}^{2}\right)$ and

$$
\operatorname{div} \vec{n}=\sum_{a=1}^{n-1} k_{a}+\mathcal{O}\left(y_{a}\right)
$$

A nearby surface $x_{n}=\epsilon$ can be obtained by taking $\vec{y} \longrightarrow \vec{y}^{\prime}=\vec{y}+\epsilon \vec{n}$ and $d y_{a}^{\prime}=d y_{a}\left(1+\epsilon k_{a}+\mathcal{O}\left(y_{a}\right)\right)$. It yields $\frac{d S^{\prime}}{d S}=\frac{\prod_{a=1}^{n-1}\left(1+\mathcal{O}\left(y_{a}^{\prime 2}\right)\right) d y_{a}^{\prime}}{\prod_{a=1}^{n-1}\left(1+\mathcal{O}\left(y_{a}^{2}\right)\right) d y_{a}}=\prod_{a=1}^{n-1}\left(1+\epsilon k_{a}\right)+$ $\mathcal{O}\left(y_{a}\right)$ near the point $\vec{y}=0$. At the line $y_{a}=0 \quad \forall a=1, \ldots, n-1$ one has

$$
\frac{d S^{\prime}}{d S}=1+\epsilon \sum_{a=1}^{n-1} k_{a}+\frac{1}{2} \epsilon^{2}\left(\left(\sum_{a=1}^{n-1} k_{a}\right)^{2}-\sum_{a=1}^{n-1} k_{a}^{2}\right)+\mathcal{O}\left(\epsilon^{3}\right) .
$$

Clearly, the relation (20) is valid for every point of the surface with its own principal curvatures. Following [6, 7] we introduce a new wave function

$$
\chi(x)=\Psi(x) \sqrt{\frac{d S^{\prime}}{d S}} .
$$

Physically it amounts to

$$
\int_{\left|x_{n}\right| \leq \delta} d V|\Psi(x)|^{2}=\int_{-\delta}^{\delta} d x_{n} \int d S|\chi(x)|^{2}
$$

so that the function $\int d x_{n}|\chi(x)|^{2}$ defines the probability density of finding the particle at a given point on the surface. For the lowest energy solution the normal motion gives only the factor of $\cos \frac{\pi x_{n}}{2 \delta}$, and we easily get [2]

$$
\tilde{\Delta} \Psi(x)=\Delta_{L B} \chi(x)+\partial_{n}^{2} \chi(x)+\left(\frac{1}{2} \sum_{a=1}^{n-1} k_{a}^{2}-\frac{1}{4}\left(\sum_{a=1}^{n-1} k_{a}\right)^{2}\right) \chi(x)+\mathcal{O}\left(x_{n}\right) .
$$


At this energy level $\chi\left(x_{1}, \ldots, x_{n}\right)=f\left(x_{1}, \ldots, x_{n-1}\right) \cos \frac{\pi x_{n}}{2 \delta}$. After taking $\delta \rightarrow 0$ limit and subtracting an infinite (proportional to $1 / \delta^{2}$ ) energy we obtain the Hamiltonian

$$
\hat{H}=-\frac{\hbar^{2}}{2} \Delta_{L B}+\frac{\hbar^{2}}{8}\left(\left(\sum_{a=1}^{n-1} k_{a}\right)^{2}-2 \sum_{a=1}^{n-1} k_{a}^{2}\right)
$$

which contains the quantum potential

$$
V_{q}=\frac{\hbar^{2}}{8}\left(\left(\sum_{a=1}^{n-1} k_{a}\right)^{2}-2 \sum_{a=1}^{n-1} k_{a}^{2}\right) .
$$

For 2-dimensional surfaces in $\mathbb{R}^{3}$ the result of da Costa [7, $V_{q}=-\frac{\hbar^{2}}{8}\left(k_{1}-k_{2}\right)^{2}$, is reproduced; for spheres $k_{a}=\frac{1}{R}$ and the potential is $V_{q}=\frac{\hbar^{2}(n-1)(n-3)}{8 R^{2}}$. If we would use a layer of varying width, some additional effective forces will appear [8.

\subsection{Some remarks and variations}

We could use an appropriate confining potential instead of infinite walls. It would lead to the lowest energy level function of the potential $V_{\text {conf }}\left(\frac{x_{n}}{\delta}\right)$ instead of $\cos \frac{\pi x_{n}}{2 \delta}$ and to another infinite energy. Note that we can also embed one curved space into another curved space [8]. Moreover, this approach can be used for the quantum graphs theory [25]. And it is currently used to describe a motion of electrons in nanostructures [26, 27] and for the physics of molecules [8, 28. Even before the general consideration of the problem appeared in [6] and [7, some elements of the thin layer approach were successfully used in the theory of chemical reactions [29]. Recently, a considerable progress in the method has been achieved [41] allowing one to treat a very general type of quantum constrained motion, even with relatively large kinetic energies, with a full mathematical rigour.

We should mention that there is one more method of quantization proposed by Prokhorov [30. The motion of a particle is considered as a system with two second class constraints but only one condition is imposed on the physical sector: $\hat{P}_{n} \Psi_{\text {phys }}(x)=0$ with $\hat{P}_{n}=-i \hbar \frac{1}{\tilde{g}^{1 / 4}} \frac{\partial}{\partial x_{n}} \tilde{g}^{1 / 4}$. It means that

$$
\partial_{n}\left(\sqrt{\frac{d S^{\prime}}{d S}} \Psi_{\text {phys }}(x)\right)=0 .
$$

Having solved some task by this method, one should put $x_{n}=0$ in the results after all the differentiations over $x_{n}$ are performed. Due to (22) the probability to find a particle at the distance $\left|x_{n}\right|$ from the surface does not depend on the value of $x_{n}$, and we choose one value we need. (For Prokhorov's view see [30.) This method gives the same results [2] as the thin layer approach due to a very 
simple physical reason. The lowest energy level wave functions (in the model with two infinite potential walls) have nodes at $x_{n}= \pm \delta$ and the bunch at $x_{n}=0: \partial_{n} \chi=0$ or, equivalently, $\hat{P}_{n} \Psi=0$.

One also could be tempted to use the Hamiltonian in curvilinear coordinates $H=\frac{1}{2}\left(\sum_{i=1}^{n-1} \frac{p_{i}^{2}}{h_{i}(x)}+p_{n}^{2}\right)$ with a simple recipe $p_{i} \longrightarrow \hat{p}_{i}=-i \hbar \tilde{g}^{-1 / 4} \partial_{i} \tilde{g}^{1 / 4}$ followed by the thin layer method. Then the result [31] for $\psi\left(x_{1}, \ldots, x_{n}\right)=$ $f\left(x_{1}, \ldots, x_{n-1}\right) \cdot \cos \frac{\pi x_{n}}{2 \delta}$

$$
\hat{H} \frac{f \cdot \cos \frac{\pi x_{n}}{2 \delta}}{\sqrt{\frac{d S^{\prime}}{d S}}}=\frac{1}{2} \sum_{i=1}^{n-1} \frac{\hat{p}_{i}^{2}}{h_{i}(x)} \frac{f \cdot \cos \frac{\pi x_{n}}{2 \delta}}{\sqrt{\frac{d S^{\prime}}{d S}}}-\frac{1}{2} \frac{\hbar^{2} f}{\sqrt{\frac{d S^{\prime}}{d S}}} \partial_{n}^{2} \cos \frac{\pi x_{n}}{2 \delta}
$$

can be considered as zero quantum potential. However, one should remember that quantization in curvilinear coordinates is dangerous because its results usually depend on the choice of coordinate system. And the curvilinear momenta operators are only symmetric but not in general selfadjoint. And what is more important, the operator ordering problem in $\frac{\hat{p}_{i}^{2}}{h_{i}(x)}$ terms is not solved. It is not difficult to deduce the correct ordering for the zero potential theory, but this particular ordering is not so natural a priori and can involve quite bulky expressions [2]. (See also [32].)

\subsection{Higher codimensions and gauge structures}

In general we can represent a smooth $m$-dimensional surface in $\mathbb{R}^{n}$ by its tangent paraboloid at a chosen point:

$$
y_{\alpha}=\frac{1}{2} \sum_{a=1}^{m} \sum_{b=1}^{m} k_{a b}^{(\alpha)} y_{a} y_{b}+\mathcal{O}\left(y_{a}^{3}\right)
$$

$\alpha=m+1, \ldots, n$ with some "curvature coefficients" $k_{a b}^{(\alpha)}=k_{b a}^{(\alpha)}$. But it turns out that this approach is convenient only for quantization on curves (and for some other relatively trivial cases like flat 2-torus in $\mathbb{R}^{4}$ ).

\subsubsection{Quantization on curves}

For 1-dimensional manifolds (curves) a suitable rotation in the space of $y_{\alpha}$ casts (23) to the form $y_{2}=\frac{1}{2} k y_{1}^{2}+\mathcal{O}\left(y_{1}^{3}\right) ; y_{3}, \ldots, y_{n}=\mathcal{O}\left(y_{1}^{3}\right)$. The unit normal vectors are $n_{1}^{(2)}=k y_{1}+\mathcal{O}\left(y_{1}^{2}\right), n_{2}^{(2)}=-1+\mathcal{O}\left(y_{1}^{2}\right), n_{3}^{(2)}=\ldots=n_{n}^{(2)}=\mathcal{O}\left(y_{1}^{2}\right)$; $n_{i}^{(\alpha)}=-\delta_{i \alpha}+\mathcal{O}\left(y_{1}^{2}\right)$ for $\alpha \geq 3$. We have $\vec{n}^{(\alpha)} \vec{n}^{(\beta)}=\delta_{\alpha \beta}+\mathcal{O}\left(y_{1}^{2}\right)$, and after the transformation $\vec{y} \rightarrow \vec{y}^{\prime}=\vec{y}+\sum_{\alpha=2}^{n} \epsilon_{\alpha} \vec{n}^{(\alpha)}$ one gets $d y_{1}^{\prime}=\left(1+\epsilon_{2} k+\mathcal{O}\left(y_{1}\right)\right) d y_{1}$ and $d y_{\alpha}^{\prime}=\left(1+\mathcal{O}\left(y_{1}\right)\right) d y_{\alpha}$ for $\alpha \geq 3$. We introduce a new curvilinear coordinate system near the curve in which $x_{1}$ is just the length along the curve and the hypersurfaces of constant $x_{1}$ are the cross sections of its tubular neighbourhood. 
And in a given cross section any point $\vec{r}$ has other $n-1$ coordinates defined by $x_{\alpha}=\vec{n}^{(\alpha)} \cdot \vec{r}$. In this coordinate system

$$
\tilde{g}_{i k}=\left(\begin{array}{cc}
\left(1+x_{2} k\right)^{2} & 0 \\
0 & I
\end{array}\right)
$$

and $\tilde{\Delta}=\Delta_{c}+\Delta_{n}+\left(\frac{1}{1+x_{2} k} \partial_{2}\left(1+x_{2} k\right)\right) \partial_{2}=\Delta_{c}+\Delta_{n}+\frac{k}{1+x_{2} k} \partial_{2}$ where $\Delta_{c}$ is Laplace-Beltrami operator on a curve $x_{\alpha}=$ const and $\Delta_{n}=\sum_{\alpha=2}^{n} \partial_{\alpha}^{2}$ is Laplace operator in a hyperplane $x_{1}=$ const. Then for a wave function $\chi(x)=\sqrt{1+x_{2} k} \Psi(x)$ in a thin layer $\sum_{\alpha=2}^{n} x_{\alpha}^{2} \leqslant \delta^{2}$ we obtain [2]

$$
\tilde{\Delta} \Psi(x)=\tilde{\Delta} \frac{\chi(x)}{\sqrt{1+x_{2} k}}=\Delta_{c} \chi(x)+\Delta_{n} \chi(x)+\frac{k^{2}}{4} \chi(x)+\mathcal{O}\left(x_{\alpha}\right) .
$$

After subtracting an infinite energy due to $\Delta_{n} \chi(x)$ it yields the quantum potential $V_{q}=-\frac{\hbar^{2}}{8} k^{2}$ as in 7 . At this point we can also see that the higher codimensional thin layer problem is not reducible to a step-by-step decreasing of the physical space dimension. Indeed, for a straight line in $\mathbb{R}^{3}$ we obviously have zero quantum potential. But if we first consider a cylinder of radius $R$ and then restrict it further to the line, the quantum potential would be $-\frac{\hbar^{2}}{8 R^{2}}$.

There is one subtlety in the above discussion. If the curve has a torsion, our coordinate system rotates around it. If one attempts at describing this motion in a non-rotating coordinate system he gets new terms in the Hamiltonian which correspond to rotations around the curve [33. Locally these descriptions are equivalent, but globally for a closed curve the rotating coordinate system may not exist and one can get global phases out of it [33. (Global effects may also be relevant for nontrivial normal bundles, for example for a motion on the Möbius strip, 24.) Note that in our consideration we need either to use a thin tube with spherical cross sections or to rotate the cross section around the curve together with its Frenet frame. For more general discussion of twisting the confining potential see [8].

\subsubsection{Higher dimensions and geometry of normal bundles}

In general we can try to use a similar construction; but such coordinate systems which eliminate all the rotations from the Hamiltonian do not exist for submanifolds with non-flat normal bundles. It was first noticed by da Costa 34 who proved that it is not always possible to find a smooth family of normal vectors with the properties required for separation of normal and tangential motions. Geometrically we need to understand the structure of a tubular neighbourhood of the physical submanifold which is naturally related to a small portion of the normal bundle corresponding to its embedding into the ambient space. Then one has to define the standard mathematical notion of the normal connection in the normal bundle $[8,35$. We will not discuss it here in any detail, but basically it amounts to taking the normal projections of the ambient space covariant 
derivatives. From the ambient (Euclidean) space viewpoint parallel transports according to this connection involve rotations including those which go around the physical subspace. For example, in the tubular neighbourhood of a curve the normal connection rotates the normal vectors not only with the normal hyperplanes but also around the curve together with its Frenet frame. Of course, on a curve we can exclude this effect by a suitable choice of rotating coordinates 2 as in the previous subsubsection. But for higher dimensional (and codimensional) manifolds the normal bundle can be non-flat and such exclusion would be impossible. The simplest examples are the configuration space of the double pendulum [34] and the helical surface from [23].

Analytically it means that the Laplace operator necessarily contains terms with mixed normal and tangential derivatives, $\partial_{a \alpha}^{2}$. They vanish at the physical surface linearly with the distance from it, bit it is obviously not enough for the thin layer approach (unlike the Prokhorov method). Da Costa concluded 34] that the thin layer quantization would not work well in this situation. This statement was repeated in 2]. Strictly speaking, this attitude is not right because all the dangerous terms sum up to the angular momenta operators corresponding to rotations in normal sections of the thin tube [8, 23, 39, 40. In the case of the lowest energy solutions for a thin tube with a spherical cross section it will not influence the resulting theory at all. But if the chosen normal energy level is degenerate then a gauge structure will show up [8, 23, 36, 37, 39, 40. The simplest example (although not very natural from the thin layer quantization viewpoint) is a higher energy level of normal motion in the thin tube 23. In any case, the quantum potential can be calculated explicitly [8, 23] but these complicated expressions do not give much to our intuition and we omit them here. (One can attempt at making the calculations by means of the tangent paraboloid technique [2] which was so useful above, but in the general case more powerful geometric methods [8, 23] are more safe and easier to implement.) Note that the Prokhorov method [30] can give the quantum potential too [2] but it is completely insensitive to the gauge structures. And let us finally mention that gauge structures (different from above) appear also in the algebraic approaches to quantization on a coset space [38; we do not discuss it here.

\section{Conclusions}

We presented the main approaches to quantization of systems with second class constraints which do not involve path integrals: redefinition of the Poisson structure (Dirac brackets), conversion of the constraints into the first class ones by introduction of new degrees of freedom and the thin tube approximation identical to a way of realizing the holonomic constraints in classical mechanics. These methods give different results but often they involve very similar geometric constructions and conditions. Many of the aspects of theory deserve a better understanding. Geometric properties of the Abelian conversion are still unclear. And it's worth trying to describe the main features of higher codimensional Dirac quantization from an explicit operatorial perspective. This investigation can 
be very important for our understanding of constrained quantum mechanics in general.

A special remark is in order concerning the reference list. We do not attempt at making it absolutely complete. But, together with the references in the references, it should suffice to give a more-or-less full picture of research in the subject, at least as much as we were able to find it out in the literature. We think it is quite important because many similar results are scattered in different works being completely disconnected from each other. Jensen and Koppe 6] didn't know about the article of Marcus [29], and neither of these works has influenced the results of da Costa [7. The second article by da Costa 34 is rarely cited (as well as the pioneering one due to Marcus 29]). The authors of [10] and [1] were unaware of earlier works [5] and [13. And in our articles [1, 2] we didn't refer to very important works [5, 8, 13, [28, 29, 33] due to the lack of knowledge.

Acknowledgements. The Author is greatful to the Cluster of Excellence EXC 153 "Origin and Structure of the Universe" for partial support; to Prof. Lev Prokhorov for pointing at the problems of quantization with second class constraints when the Author was yet a student; to Prof. Kanji Fujii for very useful e-mail correspondences concerning his articles on quantization problems; to the Organizers of the 40th Symposium on Mathematical Physics for the opportunity to participate in this wonderful conference and to give a talk; to Prof. Julio Guerrero for the interesting discussion at the Symposium.

\section{References}

[1] Golovnev A., Int. J. Geom. Meth. Mod. Phys. 3, 655 (2006); arXiv: quant-ph/0508044

[2] Golovnev A., J. Math. Phys. 47, 082105 (2006); arXiv: quant-ph/0508111

[3] Podolsky B., Phys. Rev. 32, 812 (1928).

[4] Aldaya V., Calixto M., Guerrero J., Lopez-Ruiz F.F., arXiv: 0807.2630

[5] Ogawa N., Fujii K, Kobushkin A., Progress Theor. Phys. 83, 894 (1990).

[6] Jensen H., Koppe H., Ann. Phys. 63, 586 (1971).

[7] da Costa R.C.T., Phys. Rev. A 23, 1982 (1981).

[8] Mitchell K.A., Phys. Rev. A 63, 042112 (2001); arXiv: quant-ph/0001059

[9] Dirac P.A.M., Lectures on quantum mechanics (Yeshiva University, N.Y., 1964).

[10] Kleinert H., Shabanov S.V., Phys. Lett. A 232, 327 (1997); arXiv: quant-ph/9702006 
[11] Klauder J.R., Shabanov S.V., Nucl. Phys. B 511, 713 (1998); arXiv: hep-th/9702102

[12] Grundling H., Hurst C.A., J. Math. Phys. 39, 3091 (1998); arXiv: hep-th/9712052

[13] Ogawa N., Fujii K, Chepilko N., Kobushkin A., Progress Theor. Phys. 85, 1189 (1991).

[14] Faddeev L.D., Shatashvili S.L., Phys. Lett. B 167, 225 (1986).

[15] Polyakov A. Phys. Lett. B 103, 207 (1981).

[16] Collins P.A., Tucker R.W., Nucl. Phys. B 112, 150 (1976).

[17] Marquard U., Scholl M., Phys. Lett. B 227, 227 (1989).

[18] Bars I., Nucl. Phys. B 343, 398 (1990).

[19] Hayakawa M., Ishibashi N., Nucl. Phys. B 614, 171 (2001); arXiv: hep-th/0107103

[20] Klauder J.R., Lect. Notes Phys. 572, 143 (2001); arXiv: hep-th/0003297

[21] Klauder J.R., J. Math. Phys. 40, 5860 (1999); arXiv: gr-qc/9906013

[22] Batalin I.A., Fradkin E.S., Nucl. Phys. B 279, 514 (1987).

[23] Schuster P.C., Jaffe R.L., Ann. Phys. 307, 132 (2003); arXiv: hep-th/0302216

[24] Froese R., Herbst I., Comm. Math. Phys. 220, 489 (2001); arXiv: math-ph/9909007

[25] Dell'Antonio G., Tenuta L., J. Math. Phys. 47, 072102 (2006); arXiv: math-ph/0603044

[26] Encinosa M., Etemadi B., Phys. Rev. A 58, 77 (1998).

[27] Encinosa M., Mott L., Etemadi B., Phys. Scr. 72, 13 (2005); arXiv: quant-ph/0409141

[28] Maraner P., Ann. Phys. 246, 325 (1996); arXiv: hep-th/9406004

[29] Marcus R.A., J. Chem. Phys. 45, 4493 (1966).

[30] Nuramatov A.G., Prokhorov L.V., Int. J. Geom. Meth. Mod. Phys. 3, 1459 (2006); arXiv: quant-ph/0507038 
[31] Encinosa M., arXiv: quant-ph/0508104

[32] Liu Q.H., Tong C.L., Lai M.M., J. Phys. A 40, 4161 (2007).

[33] Takagi S., Tanzawa T., Progress Theor. Phys. 87, 561 (1992).

[34] da Costa R.C.T., Phys. Rev. A 25, 2893 (1982).

[35] Lin C., Lu Z., Comm. Part. Diff. Eq. 31, 1529 (2006);

arXiv: math/0601201 (math.DG)

[36] Fujii K., Ogawa N., Progress Theor. Phys. 89, 575 (1993).

[37] Fujii K., Ogawa N., Uchiyama S., Chepilko N.M., Int. J. Mod. Phys. A 12, 5235 (1997).

[38] McMillan D., Tsutsui I., Ann. Phys. 237, 269 (1995).

[39] 1 Maraner P., Destri C., Mod. Phys. Lett. A 8, 861 (1993).

[40] Maraner P., J. Phys. A 28, 2939 (1995); arXiv: hep-th/9409080

[41] S. Teufel, J. Wachsmuth, in preparation.

\footnotetext{
${ }^{1}$ The last three references are added in the second version. They are placed in the end of the reference list in order to keep the other numbers intact.
} 